\title{
Value of bronchial wash and brush cytology in addition to histology in the diagnosis of lung cancer
}

\author{
Ruchee Khandelwal $^{1}$, Deepti Agarwal ${ }^{2, *}$, Surbhi Bansal ${ }^{3}$ \\ ${ }^{1}$ Associate Professor, ${ }^{2}$ Assistant Professor, ${ }^{3}$ Post Graduate, Dept. of Pathology, Shri Ram Murti Smarak Institute of Medical \\ College, Bhojipura, Bareilly, Uttar Pradesh, India
}

*Corresponding Author:

Email: dr.deeptiagarwal86@gmail.com

Received: $9^{\text {th }}$ February, 2018

Accepted: $19^{\text {th }}$ February, 2018

\begin{abstract}
A retrospective study was performed to evaluate the diagnostic yield of lung cancer on biopsy, bronchial washings and brushings taken at fibreoptic bronchoscopy. Records of 526 patients were analysed and eventually 107 patients with endobronchial lesions, who had had biopsy, washing and brushing were included in this study. 89 cases were found to be malignant. Squamous cell carcinoma was the most common malignancy. Brush was found to be better than wash in tumor typing, though overall tumor typing using cytology was poor. Sensitivities of bronchial wash, bronchial brush and biopsy were found to be $70.8 \%, 64 \%$ and $94.4 \%$ respectively. Biopsy gave the only positive result in $38.2 \%$, only brush in $1.1 \%$ and only wash in $1.1 \%$ malignant cases. Diagnostic accuracy of bronchial biopsy, brush and wash was found to be $95.3 \%, 70 \%$ and $73.8 \%$ respectively. Addition of bronchial wash and brush increased the positive yield by only $2.2 \%$ which was insignificant. It was concluded that the wash and brush cytology do not significantly add to the overall yield of positive results obtained from biopsies in endobronchial lesions.
\end{abstract}

Keywords: Lung cancer, Fibreoptic bronchoscopy, Bronchial wash cytology, Bronchial brush, Endobronchial biopsy, Diagnostic accuracy.

\section{Introduction}

Lung cancer has been the most common cancer in the world for several decades. It is still the most common cancer in men worldwide. In females the incidence rates are generally lower, but worldwide lung cancer is now the fourth most frequent cancer of women and the second most common cause of death from cancer. ${ }^{1}$ The increase in the incidence of lung cancer follows the increasing adoption of cigarette smoking. Bidi smoking appears to carry a higher lung cancer risk. ${ }^{1}$ Other risk factors implicated are air pollution, radioacitivity and occupational exposure to asbestos, arsenic and its compounds, chromates, polycyclic aromatic hydrocarbons and certain nickel bearing dusts. ${ }^{1}$

The original approach to the early detection was based on examination of conventional chest roentgenogram and sputum cytology. More sophisticated imaging techniques such as spiral CT are now proposed to screen for the very earliest, potentially curable cancers. $^{2}$

The advent of fibreoptic endoscopic technique in the 1960s has changed the practice of respiratory tract cytology. ${ }^{3}$ A variety of cytological specimens that include bronchial aspirates, washings, bronchial brushings and bronchoalveolar lavage can now be obtained for making the diagnosis. Transbronchial FNA biopsy can also be performed during fibreoptic bronchoscopy, thus obviating the need for open biopsy. ${ }^{4}$

Several studies have been done to evaluate the sensitivity of bronchoscopic brushings and washings.
Some studies document $90 \%$ sensitivity for central lesions and $70 \%$ for peripheral lesions ${ }^{3}$ whereas others report much lower values. Histological or cytological confirmation is known to be associated with a higher rate of specific oncology treatment for lung cancer patients. ${ }^{5} \mathrm{~A}$ negative result by any single diagnostic method cannot exclude malignancy; however using a selection or combination of these diagnostic methods upto $98 \%$ central and $94 \%$ of peripheral lung tumors may be diagnosed pre therapeutically. ${ }^{3}$

In our study we evaluated the diagnostic yield from cytology and histology in cases where a suspected endobronchial lesion was seen at flexible bronchoscopy and the results from the two modalities were compared to find out the best combination of tests to diagnose lung cancer in our set up.

\section{Materials and Methods}

This is a 2 year retrospective study conducted in the department of Pathology at Shri Ram Murti Smarak Institute of Medical Sciences, Bareilly. The records of 526 patients who underwent bronchoscopy on grounds of clinic-radiological suspicion of lung cancer during two year period from August 2015 to July 2017 were analyzed. 107 patients with endobronchial lesions suspicious of cancer on bronchoscopy in whom all the three tests including bronchial biopsy, brush and wash cytology have been done were included in the study and others were excluded.

The samples were obtained by Flexible Fibre Optic bronchoscopy done by a well experienced pulmonologist in the following order: 
Bronchial Brushings: Brushings were done using a standard brushing catheter. The specimens were smeared onto a glass slide in a circular motion. Smears were stained with Papanicolaou and MGG stains.

Endobronchial Biopsies: Biopsy specimens were taken from the suspected abnormal areas. The tissues were fixed in $10 \%$ neutral buffered formalin. These were processed in automatic tissue processor. Later on paraffin embedded blocks were prepared and thin sections of 4-5 micron thickness were cut and stained with hematoxylin and eosin.

Bronchial Washings: Washings were carried out by flushing the tumor with $20-40 \mathrm{ml}$ of $0.9 \%$ normal saline. The aspirated fluid was centrifuged and cytocentrifuged and the smears were stained with MGG and Papanicolaou stains.

The results were broadly categorized into malignant, benign, suspicious or atypical and unsatisfactory/inadequate. The malignant cases were further classified as Squamous cell carcinoma, Adenocarcinoma, Small cell carcinoma or any other specific type, ${ }^{6}$ wherever possible. Those cases in which cytological distinction between squamous cell carcinoma and adenocarcinoma was not possible, were classified under Non-small cell carcinoma and those where further tumor typing could not be done at all were classified simply as positive for malignancy.

For both cytology and histology only specimens with unequivocal malignant features, were considered to be positive. Unsatisfactory and inadequate smears were those which showed poor cellularity, degenerated cells, too much blood or necrotic debris.

Taking into consideration that no single negative test can exclude malignancy, all the cases which were unequivocally positive on any test were considered malignant (True positives). All the suspicious/atpical cases on cytology which were later proved to be malignant on histopathology were also included in this category. True negatives were the cases which were both cytologically and histologically benign. Any case with an atypical cytodiagnosis which on histopathology turned out to be benign was labeled as false positive. False negative was a case diagnosed benign on cytology but later on histopathology turned out to be malignant.

The sensitivity, specificity, diagnostic accuracy, positive and negative predictive values were calculated for each test by utilizing the predictive value model of Galen and Gambino. ${ }^{7}$

\section{Results}

107 cases of endoscopically visible lesions who underwent endobronchial biopsy, brushing and washing were included in the study. The age of the patients ranged from 32-89 years. Cough and expectoration were the most common presenting symptoms followed by chest pain, hemoptysis and weight loss.

Table 1 elaborates the broad categorization of different specimens on microscopic examination.
All 37 cases diagnosed as positive for malignancy on wash and 38 cases on brush were confirmed to be positive on biopsy and were included in true positives. Out of 28 cases diagnosed as atypical or suspicious for malignancy on wash; 26 were confirmed for malignancy on biopsy and were included in true positives whereas 2 cases revealed metaplastic changes with hyperplasia and dysplasia. These two cases were included in false positive. The wash cytology was reported as benign in 37 cases and was inadequate in 5 cases which were together included in negative test result category. Out of these 42 cases, only 16 were true negative cases. All 19 cases diagnosed as atypical or suspicious for malignancy on brush were confirmed for malignancy on biopsy and were included in true positives. There were no false positive cases on brush cytology. The brush cytology was reported as benign in 46 cases and as inadequate in 4 cases which were included together in negative test result category. Out of these 50 cases, 18 were true negatives and 32 were false negative. (Table 2)

On endobronchial biopsy 84 cases were diagnosed as malignant, 17 cases as benign, 6 cases as inadequate or inconclusive. Granulomas were found in 4 out of 17 cases diagnosed as benign whereas 11 cases revealed chronic non-specific inflammation and 2 revealed hyperplasia of epithelium with dysplasia and 2 revealed metaplasia of lining epithelium. However, 2 cases with features of chronic non- specific inflammation revealed malignancy on brush/wash that were confirmed by CT guided FNAC and were considered false negative. CT guided FNAC was performed in six cases with inadequate biopsy and in four cases with benign biopsy due to strong clinical suspicion. Three cases out of these 10 cases were found to be malignant on FNAC. Thus in our study a total of $89(83.2 \%)$ cases were malignant and $18(16.8 \%)$ were benign.

One case diagnosed as squamous cell carcinoma on wash was found to be adenocarcinoma on biopsy. In one case of small cell carcinoma brush cytology was found even better than biopsy in tumor typing. It was later confirmed by Immunohistochemistry. However, 2 cases which were diagnosed as non small cell carcinoma on brush cytology were found to be small cell carcinoma on biopsy. 1 case diagnosed as adenosqumaous carcinoma on biopsy was diagnosed as squamous cell carcinoma on both brush and wash. Interestingly the case of Adenoid cystic carcinoma could be diagnosed accurately on all the three techniques. (Table 3)

Out of 89 positive cases, only biopsy was positive in 34 cases, only brush in one case and only wash in one case.

Majority of the positive cases were found in males with male (68): female (21) ratio of 3.2:1. Peak age incidence was found in 6 decade followed by 7 decade (Table 4). 
The commonest tumor type was Squamous cell carcinoma (45\%) (Fig. 1 and 2), followed by small cell carcinoma (18\%) (Fig. 3 and 4) and adenocarcinoma (16.9\%) (Fig. 5 and 6). Among men, squamous cell carcinoma was the commonest malignancy whereas in females adenocarcinoma was most common (Table 5).

Table 6 compares the sensitivity, specificity, positive predictive value, negative predictive value and diagnostic accuracy for bronchial brush, wash and biopsy. Bronchial biopsy was found to be the best screening test with highest diagnostic accuracy (95.3\%). The addition of bronchial wash and brush to endobronchial biopsy increased the positive yield from $94.38 \%$ to $96.62 \%$.

Table 1: Broad categorization of lung lesions on cytological and histopathological examination

\begin{tabular}{|l|c|c|c|}
\hline \multicolumn{1}{|c|}{ Method } & Bronchial wash & Bronchial brush & Bronchial biopsy \\
\hline Benign & 37 & 46 & 17 \\
& $(34.6 \%)$ & $(43 \%)$ & $(15.9 \%)$ \\
\hline Atypical/suspicious & 28 & 19 & 00 \\
& $(26.1 \%)$ & $(17.8 \%)$ & $(00)$ \\
\hline Malignant & 37 & 38 & 84 \\
& $(34.6 \%)$ & $(35.5 \%)$ & $(78.5 \%)$ \\
\hline Inadequate & 05 & 04 & 06 \\
& $(4.7 \%)$ & $(3.7 \%)$ & $(5.6 \%)$ \\
\hline
\end{tabular}

Table 2: Test results of various techniques used

\begin{tabular}{|l|c|c|c|c|}
\hline \multicolumn{1}{|c|}{ Method } & $\begin{array}{c}\text { True Positive } \\
\text { cases }\end{array}$ & $\begin{array}{c}\text { False Positive } \\
\text { cases }\end{array}$ & $\begin{array}{c}\text { True Negative } \\
\text { cases }\end{array}$ & $\begin{array}{c}\text { False Negative } \\
\text { cases }\end{array}$ \\
\hline Bronchial wash & 63 & 02 & 16 & 26 \\
\hline Bronchial brush & 57 & 00 & 18 & 32 \\
\hline Bronchial biopsy & 84 & 00 & 18 & 05 \\
\hline
\end{tabular}

Table 3: Elaborates the tumor typing done in positive cases on bronchial brush, wash and biopsy

\begin{tabular}{|l|c|c|c|}
\hline \multicolumn{1}{|c|}{ Tumor type } & Bronchial wash & Bronchial brush & Bronchial biopsy \\
\hline Squamous cell carcinoma & 06 & 07 & 39 \\
\hline Adenocarcinoma & 04 & 08 & 15 \\
\hline Small cell carcinoma & 02 & 04 & 16 \\
\hline Non-small cell carcinoma & 13 & 12 & 09 \\
\hline Large cell carcinoma & 00 & 00 & 01 \\
\hline Adenosuamous carcinoma & 00 & 00 & 01 \\
\hline Adenoid cystic carcinoma & 01 & 01 & 01 \\
\hline Positive for malignancy & 11 & 06 & 02 \\
\hline \multicolumn{1}{|c|}{ Total } & 37 & 38 & 84 \\
\hline
\end{tabular}

Table 4: Age and sex wise distribution of lung cancers

\begin{tabular}{|l|c|c|c|c|}
\hline \multicolumn{1}{|c|}{ Age (years) } & Males & Females & Total & Percentage (\%) \\
\hline $31-40$ & 03 & 03 & 06 & 6.7 \\
\hline $41-50$ & 13 & 02 & 15 & 16.9 \\
\hline $51-60$ & 21 & 08 & 29 & 32.6 \\
\hline $61-70$ & 21 & 06 & 27 & 30.3 \\
\hline$>70$ & 10 & 02 & 12 & 13.5 \\
\hline Total & 68 & 21 & 89 & 100 \\
\hline
\end{tabular}

Table 5: Frequency of different types of tumors in males and females

\begin{tabular}{|l|c|c|c|c|}
\hline \multicolumn{1}{|c|}{ Tumor type } & Males & Females & Total (89) & Percentage (\%) \\
\hline Squamous cell carcinoma & 34 & 6 & 40 & 45 \\
\hline Small cell carcinoma & 11 & 5 & 16 & 18.0 \\
\hline Adenocarcinoma & 8 & 7 & 15 & 16.9 \\
\hline Poorly differentiated Non-small cell carcinoma & 10 & 3 & 13 & 14.6 \\
\hline Large cell carcinoma & 1 & 0 & 1 & 1.1 \\
\hline Adenosuamous carcinoma & 1 & 0 & 1 & 1.1 \\
\hline
\end{tabular}




\begin{tabular}{|l|l|l|l|l|}
\hline Adenoid cystic carcinoma & 1 & 0 & 1 & 1.1 \\
\hline No tumor typing & 2 & 0 & 2 & 2.2 \\
\hline
\end{tabular}

Table 6: Comparisons of indices of various techniques

\begin{tabular}{|l|c|c|c|c|c|}
\hline \multicolumn{1}{|c|}{ Method } & $\begin{array}{c}\text { Sensitivity } \\
\mathbf{( \% )}\end{array}$ & $\begin{array}{c}\text { Specificity } \\
(\boldsymbol{\%})\end{array}$ & $\begin{array}{c}\text { Positive Predictive } \\
\text { Value (\%) }\end{array}$ & $\begin{array}{c}\text { Negative } \\
\text { Predictive value } \\
(\boldsymbol{\%})\end{array}$ & $\begin{array}{c}\text { Diagnostic } \\
\text { accuracy (\%) }\end{array}$ \\
\hline Bronchial wash & 70.8 & 88.9 & 97 & 38.1 & 73.8 \\
\hline $\begin{array}{l}\text { Bronchial } \\
\text { brush }\end{array}$ & 64.0 & 100 & 100 & 36 & 70 \\
\hline $\begin{array}{l}\text { Bronchial } \\
\text { biopsy }\end{array}$ & 94.4 & 100 & 100 & 78.3 & 95.3 \\
\hline
\end{tabular}

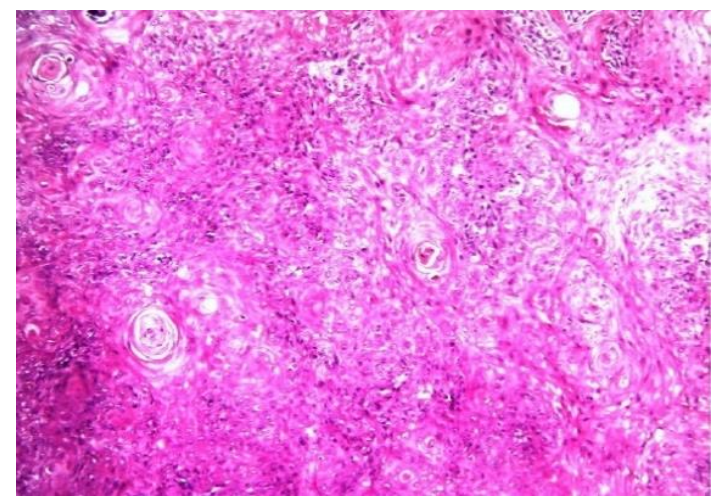

Fig. 1: Photomicrograph of bronchial biopsy showing squamous cell carcinoma (40x)

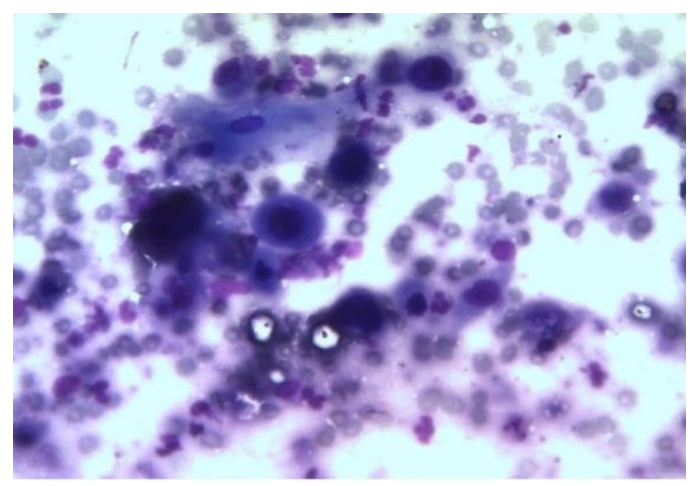

Fig. 2: Photomicrograph of cytological smear showing squamous cell carcinoma (40x)

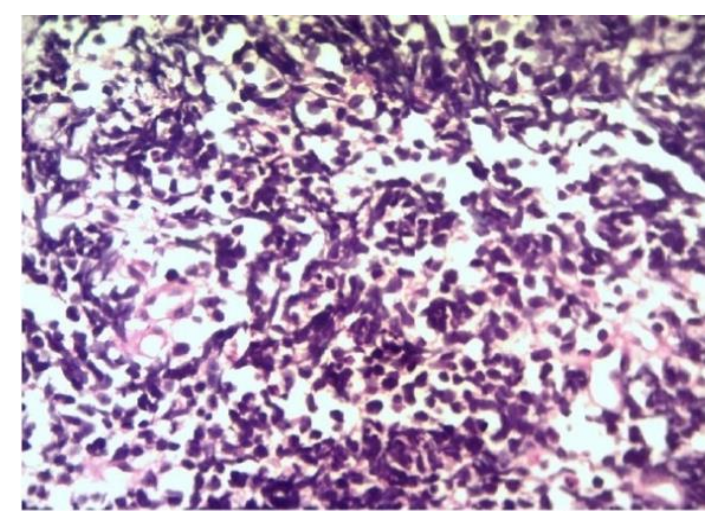

Fig. 3: Photomicrograph of bronchial biopsy showing small cell carcinoma (40x)

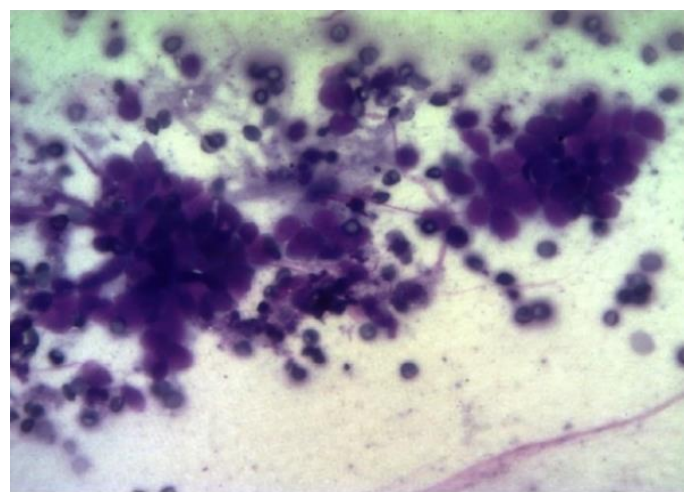

Fig. 4: Photomicrograph of cytological smear showing small cell carcinoma (40x)

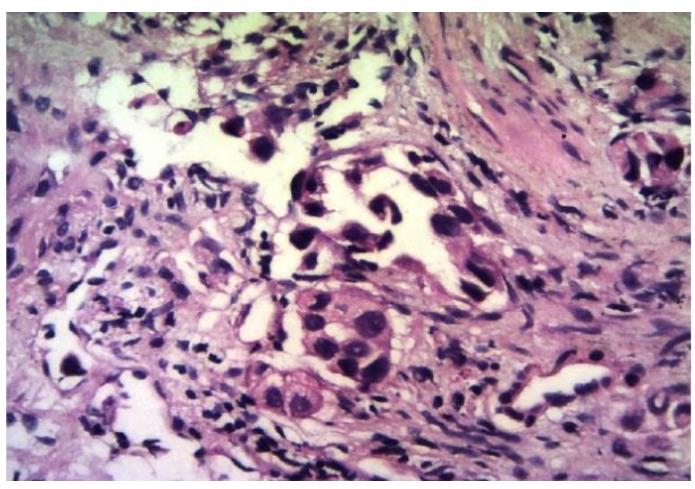

Fig. 5: Photomicrograph of bronchial biopsy showing adenocarcinoma (40x)

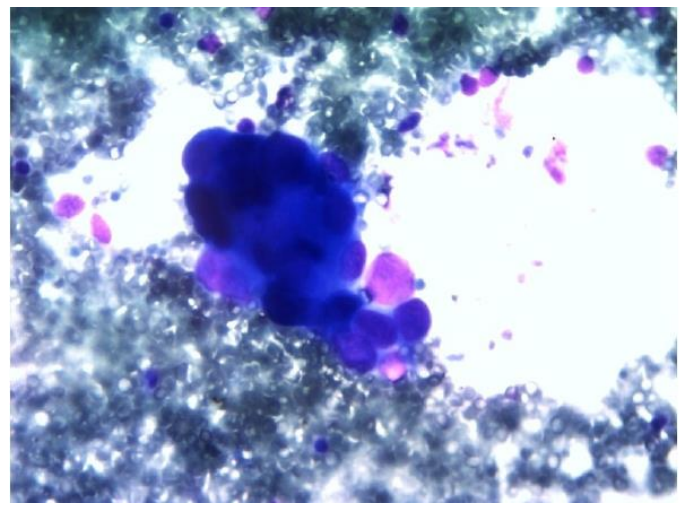

Fig. 6: Photomicrograph of cytological smear showing adenocarcinoma (40x) 


\section{Discussion}

Lung cancer mortality remains a major health issue causing over a million deaths worldwide in 2000 according to WHO data. ${ }^{8}$ Early diagnosis and treatment remain the mainstay for reducing the mortality. Pulmonary cytology and histopatholgy are valuable tools in the diagnosis of lung cancer.

107 cases with endobronchial lesions were studied to assess the sensitivity and specificity of bronchoscopic washings and brushings and comparing them with endoscopic biopsy.

In our study total 89 cases $(83 \%)$ were diagnosed as malignant by different modalities. The $\mathrm{M}$ : $\mathrm{F}$ ratio in malignant cases was 3.2:1 which is similar to that reported in several studies ${ }^{9-11}$ whereas a higher ratio has been reported by some. ${ }^{12,14}$ The peak incidence of the lung cancers was seen in 6 decade followed by 7 decade. Most of the other studies have reported 6 decade and 5 decades as commonest. ${ }^{7,9,12,13}$ Vital statistics of United states reported that majority bronchogenic carcinomas are between 55-74 years. ${ }^{9}$

Squamous cell carcinoma was the most common malignancy in our study constituting $40(44.9 \%)$ cases, followed by small cell carcinoma $(16,17.9 \%)$ cases and adenocarcinoma $(15,16.8 \%)$ which is in agreement with others ${ }^{11,14,15}$ while some have found adenocarcinoma more common than small cell carcinoma. ${ }^{12,16} \mathrm{We}$ found squamous cell carcinoma to be the most common malignant tumor in males and adenocarcinoma in females which is similar to several other studies. ${ }^{9,16}$ This is related to higher prevalence of smoking in men.

Tumor typing into specific categories could be done only in 12 out of 37 positive cases on wash (ie $35 \%$ ) and 22 out of 38 positive cases on brush (ie $57.9 \%)$. Bodh et and Lam et al have also reported higher tumor typing possibility on brush cytology as compared to bronchial wash. ${ }^{11,17}$ Problems in tumor typing on cytology have also been faced by ${ }^{13,18}$ Jones et al could type $94 \%$ cases on cytology only into broad categories of small cell and non small cell carcinoma. ${ }^{15}$ In the past, it was considered sufficient to simply differentiate between non-small cell carcinoma and small cell carcinoma, commonly possible on bronchial brushings and washings, for treament of lung cancer. However the work from Scagliotti et a ${ }^{19}$ has shifted the need from simply diagnosing non- small cell carcinoma to the need to identify the histological subtype, in order for the optimal chemotherapy regimen to be recommended for the patient. The difficulties on specifying tumors on cytology is due to lack of adequate cellularity, keratinization, and mucus production, hemodilution and inflammation.

We faced problem in tumor typing in $13.1 \%$ cases on biopsy as well which is higher than in the study done by Bodh et al (3.2\%). ${ }^{11}$ However, Reddy et al, Rawat et al and Jones et al have reported more number of nonsmall cell carcinoma on biopsy. Immunohistochemistry may be helpful in such cases. ${ }^{9}, 14,15$
We found that cytology was better in typing the tumor than histology in $4.4 \%$ cases ( 3 adenocarcinoma and 1 small cell carcinoma). Jones et al also found cytology better than biopsy in typing two cases of small cell carcinoma. ${ }^{15}$ Jones et al and Lam et al have also challenged the common assumption that when comparing histology and cytology, the final histological diagnosis is always correct, suggesting that in some cases cytological features as opposed to tissue patterns might be of more value. ${ }^{15,17}$

We observed $28(26.1 \%)$ suspicious cases on wash and $19(17.8 \%)$ on brush. Variable number of suspicious cases have been reported in different studies ranging from 3 to $12.6 \%$. $^{7,13,15,18}$ All our suspicious cases, except two, were later confirmed to be malignant on biopsy. Similar observations have also been made by Ahmad et al and Raiza et al., ${ }^{713}$ However Rao et al failed to confirm malignancy in all their suspicious cases. ${ }^{18}$ This could be due to the fact that these cases were neither followed up nor confirmed by any other test.

We observed $24.3 \%$ false negative cases on bronchial wash and $29.9 \%$ on bronchial brush. The reason for missing the lesions on cytology could be due to several factors like secondary inflammation, nonrepresentative material and suboptimal yield.

We reported only $2(1.9 \%)$ false positive cases on wash and none on bronchial brush and biopsy, so far as we can tell. Similarly low false positive rate on cytology has been observed by Ahmad et al and Raiza et al. ${ }^{7,13}$ However Bodh et al have reported higher rate. ${ }^{11}$ This is because they have taken biopsy as reference and they have later found that not all these cases were false positive especially on brush cytology. Rao et al have also observed $6.8 \%$ false positive cases. ${ }^{18}$ False positive cases may be due to reactive atypia secondary to inflammation, squamous metaplastic cells and basal cell hyperplasia. In our study we found metaplasia and hyperplasia in 2 false positive cases reported.

Bronchial biopsy was found to be false negative in $5(4.6 \%)$ cases. Bodh et al, Jones et al, Karahalli et al and Stringfield et al have also observed false negative bronchial biopsies. ${ }^{11,15,20,21}$ Bodh et al diagnosed 8 cases of adenocarcinoma on brush in biopsy negative cases, especially in compressive type of lesions on bronchoscopy. ${ }^{11}$ This could be due to rather small and shallow samples of tissue obtained with the small biopsy forceps used in this procedure. ${ }^{21}$ We reported 6 (5.6\%) biopsies as inadequate. Jones et al have reported inadequate biopsy rate to be less than $1 \%{ }^{15}$

Of the malignant cases, biopsy was positive in $94.4 \%$ cases, brush in $64 \%$ cases and wash in $70.7 \%$ cases. Some authors have found yield of biopsy to be better in endobronchial lesions than submucosal lesions ${ }^{15,17,22-28}$ and reported biopsy sensitivity between $71.6 \%$ and $97 \%$ for endobronchial lesions. Variable biopsy yields reported by different authors may be due 
to different policies about number of samples taken, definition of endobronchial lesions and expertise of the pulmonologists. It is known that maximum diagnostic yield is achieved after at least 4 to 5 samples have been taken. ${ }^{27}$

Values between $31.6 \%{ }^{20}$ and $84.8 \%{ }^{23}$ have been reported by various authors ${ }^{11,14,20,23,25,26,29}$ for wash sensitivity and between $28.5 \%{ }^{30}$ and $85.6 \%{ }^{31}$ for brush. ${ }^{11,12,14,20,25,26,30,31}$ The different values may be due to the use of different techniques for the retrieval and processing of cytological specimens, variations in the use or non use of biplanar fluoroscopy and different practices with regard to suspicious cytological appearances, which some but not all workers take as positive results in the analysis.

Several studies have been done in the past to decide the most effective combination of diagnostic techniques, in particular, the relative value of bronchial brushings and washings. In most of the studies washings offered no advantage. ${ }^{16,20,24}$ Chaudhary et al found diagnostic yield of wash to be even higher than biopsy. ${ }^{29}$ Karahalli et al and Saita et al have reported higher yield using brush cytology. ${ }^{20,31}$ Rawat et al, Jones et al., Lam et al, Stringfeild et al, Mak et al, Dobler et al, Troung et al found an increased yield of positive cases when both brush and bronchial wash were used together with biopsy..$^{14,15,17,21,25,26,32}$ Govert et al found the sensitivity of bronchoscopy increased from $80.8 \%$ to $84.8 \%$ or $85.3 \%$ when either brush or wash was added to the biopsy respectively. ${ }^{23}$ They suggested that collection of either brush or wash to be the best strategy. In our study, there was an additional yield of only $2.2 \%$ when both brush and wash were combined with biopsy which is insignificant ( $p>0.05$ ). Similarly Fauzi et al and Chechani et al have also failed to find any additional yield and have discouraged routine use of cytology. ${ }^{22,33}$

Jones et al and Mak et al suggested the idea of holding washing specimens for processing until a time when biopsy or brush results were negative in highly suspicious cases of lung cancer. ${ }^{15,25}$ This may be a more cost effective approach in reducing the pathologist load with the added advantage of occasional case which was missed on biopsy. However, a repeat biopsy may still be required if wash fails to type the tumor and besides a negative wash cannot exclude malignancy owing to low sensitivity.

One limitation in our study was that no follow up studies or any other confirmatory tests could be done on all biopsy negative cases. So, there could have been more false negative cases on biopsy than reported.

Our data shows that endobronchial biopsy is the best test to diagnose endobronchial lesions; and bronchial wash and brush cytology have no added advantage. Besides exact tumor typing required for treatment today cannot be done reliably on cytology.

\section{Conclusion}

There is no significant increase in the number of positive cases detected when bronchial washings and brushings are added to the endobronchial biopsy for diagnosing lung cancer patients. Evaluating bronchial washing and brushing specimens only when the endobronchial biopsy are negative seems the best approach. They may also help in primary screening of patients where biopsy cannot be taken.

Pathological and molecular analysis are playing an increasing role in guiding the management of patients with non-small cell lung carcinoma, thus increasing the need for larger sample of tissue for appropriate analysis and making bronchial washings and brushings increasingly inadequate.

Thus, we do not advocate regular use of bronchial wash and brush cytology in diagnosing lung cancer patients.

\section{Conflicts of Interest: None declared}

\section{References}

1. Park K. Park's textbook of preventive and social medicine. $23^{\text {rd }}$ ed. Jabalpur: M/s Banarsidas Bhanot;2015.p.390.

2. Myron RM. Tumors of the lung: Conventional Cytology and Aspiration Biopsy. In: Leopold GK, Myron RM, editors. Koss' Diagnostic and its histopathologic bases.5 ed. Philadelphia: Lippincott Williams and Wilkins; 2006.p.643-712.

3. Thomas EG, Julie M, Winifred G. Respiratory tract. In: Winifred G, Gabrijela K, editors. Diagnostic cytopathology. $3^{\text {rd }}$ ed. London: Churchill Livingstone Elsevier;2010.p.17-116.

4. Geisinger KR, Raab SS, Stanley MW, Silverman JF. Modern cytopathology. Philadelphia: Churchill Livingstone;2004.p.404-32.

5. Connolly CK, Jones WG, Thorogood J, Head C, Muers MF. Investigation, treatment and prognosis of bronchial carcinoma in the Yorkshire region of England, 19761983. Br J Cancer 1990;61:579-583.

6. Travis WD, Brambilla E, Muller-Hermelink HK, Harris CCv (Eds): World Health Organization Classification of Tumors. Pathology and genetics of Tumors of the lung, pleura, thymus and heart. IARC Press: Lyon 2004.

7. Ahmad M, Afzal S, Saeed W, Mubarik A, Saleem N, Khan SA et al. Efficacy of Bronchial wash cytology and its correlation with biopsy in lung tumors. J Pak Med Assoc. 2004 Jan;54(1):13-6.

8. Fraser GS, Bhatt N, Dangoor A, Sephton M. Classification and treatment of non-small-cell lung carcinoma. In: Pignatelli M, Gallagher P, editors. Recent Advances in Histopathology. 23 ed. London: JP Medical Ltd;2014.p.31-45.

9. Reddy S, Vivekanand N, Durga K. Efficacy of bronchial wash and brush cytology in the diagnosis of lung cancers. Sch J App Med. Sci. 2014;2(2D):816-20.

10. Liwsrisakun C, Pothirat C, Bumroongkit C, Deesomchok A. Role of Bronhchial washings in the diagnosis of endoscopically visible lung cancer. J Med Assoc Thai. 2004;87(6):599-604. 
11. Bodh A, Kaushal V, Kashyap S, Gulati A.

Cytohistological correlation in diagnosis of lung tumors by using fibreoptic bronchoscopy: Study of 200 cases. Indian J Pathol Microbiol. 2013Apr;56(2):84-8.

12. Fuladi AB, Munje RP, Tayade BO. Value of washings, brushings and biopsy at fibreoptic bronchoscopy in the diagnosis of lung cancer. J Indian Acad clin Med. 2004 Apr;5(2):137-42.

13. Raiza D, Rout S, Reddy KP, Ramalaxmi P, Prithvi BK, Harikishan KS. Efficacy of bronchial wash and brush cytology and its correlation with biopsy in lung lesions. IJHRMIMS.2014Oct;1(1):21-24.

14. Rawat J, Sindhwani G, Saini S, Kishore S, Kusum A, Sharma A. Usefulness and cost effectiveness of bronchial washing in diagnosing endobronchial malignancies. Lung India. 2007;24:139-41.

15. Jones AM, Hanson IM, Armstrong GR, O'Driscoll BR. Value and accuracy of cytology in addition to histology in the diagnosis of lung cancer at flexible bronchoscopy. Respir Med. 2001;(95):374-78.

16. Sengupta $\mathrm{M}$, Saha K. Computed tomography guided fine needle aspiration cytology of Pulmonary mass lesions in a tertiary care hospital: A two year prospective study. Med J DY Patil Univ. 2014 Mar;7(2):177-81.

17. Lam WK, So SY, Hsu C, Yu DYC. Fibreoptic bronchoscopy in the diagnosis of bronchial cancer: comparison of washings, brushings and biopsies in central and peripheral tumours. Clin Oncol. 1983;9:3542.

18. Rao S, Rao S, Lal A, Barathi G, Dhanasekar T, Duvuru P. Bronchial wash cytology: A study on morphology and morphometry. J Cytol. 2014Apr;31(2):63-7.

19. Scagliotti GV, Parikh P, Von Pawel J, et al. Phase III study comparing cisplastin plus gemcitabine with cispalstin plus pemetrexed in chemotherapy-naïve patients with advanced -stage- non-small cell lung cancer. J Clin Oncol. 2008;26:3543-3551.

20. Karahalli E, Yilmaz A, Türker H, Ozvaran K. Usefulness of various diagnostic techniques during fiberoptic bronchoscopy for endoscopically visible lung cancer: Should cytologic examinations be performed routinely? Respiration 2001;68:611-4.

21. Stringfield JT, Markowitz DJ, Bentz RR et al. The effect of tumor size and location on diagnosis by fiberoptic bronchoscopy. Chest, 1977;72(4):474-6.

22. Fauzi AR, Balakrishnan L, Rathor MY. Usefulness of cytological specimens from bronchial brushings and washings in addition to endobronchial biopsies during bronchoscopy for lung cancer: 3 years data from a chest clinic in a general hospital. Med J Malaysia. 2003Dec;58(5):729-34.

23. Govert JA, Kopita JM, Matchar D, Kussin PS, Samvelson WM. Cost-effectiveness of collecting routine cytologic specimens during routine bronchoscopy for endoscopically visible lung tumour. Chest 1996;109:451456.
24. Rosell A, Monso E, Lores L et al. Cytology of bronchial biopsy rinse fluid to improve the diagnostic yield of lung cancer. Eur Respir J. 1998;12(6):1415-8.

25. Mak VHF, Johnston IDA, Hetzel MR, Grubb C. Value of washings and brushings at fibreoptic bronchoscopy in the diagnosis of lung cancer. Thorax. 1990;45:373-376.

26. Dobler CC, Crawford AB. Bronchoscopic diagnosis of endoscopically visible lung malignancies: should cytological examinations be carried out routinely. Intern Med J. 2009 Dec;39(12):806-11.

27. Gellert AR, Mudd RM, Sinha G, Geddes DM. Fiberoptic bronchoscopy: effect of multiple bronchial biopsies on diagnostic yield in bronchial carcinoma. Thorax. 1982;37:684-687.

28. Minami H, Ando Y, Nomura F, Saki S, Shimokata K. Interbronchoscopist variability in the diagnosis of lung cancer by flexible bronchoscopy. Chest. 1994;105:16581562.

29. Chaudhary BA, Yoneda K, Burki NK. Fiberoptic bronchoscopy: comparison of procedures used in the diagnosis of lung cancer. J Thorac Cardiovasc Surg. 1978;76:33-7.

30. Soler Tv, Isamitt DD Carrasco OA. Yield of biopsy, brushing and bronchial washing through fiberbronchoscopy in the diagnosis of lung cancer with visible lesions. Rev Med Chil. 2004 oct;132(10):1198203.

31. Saita S, Tanzillo A, Riscica C, Maresca A, Potenza E, D' Arrigo M. Bronchial brushings and biopsy: a comparative evaluation in diagnosing visible bronchial lesions. Eur J Cardiothorac Surg. 1990;4(5):270-2

32. Truong LD, Underwood RD, Greenberg SD, et al. Diagnosis and typing of lung carcinomas by cytopathologic methods: a review of 108 cases. Acta Cytol. 1985;29:379-84.

33. Chechani v. Bronchoscopic diagnosis of solitary pulmonary nodules and lung masses in the absence of endobronchial abnormality. Chest. 1996;109:620-25.

How to cite this article: Khandelwal $\mathrm{R}$, Agarwal D, Bansal S. Value of bronchial wash and brush cytology in addition to histology in the diagnosis of lung cancer. Ind J Pathol Oncol, 2018;5(3):498-504. 